\title{
Expansion of vacuum magnetic fields in toroidal harmonics
}

\author{
B.Ph. van Milligen, A. Lopez Fraguas \\ Asociación EURATOM-CIEMAT para Fusión \\ Avda. Complutense 22, 28040 Madrid, Spain
}

\begin{abstract}
The vacuum magnetic field in stellarators and tokamaks is expanded in toroidal harmonics (halfinteger Legendre functions). In addition to the commonly used external harmonics (irregular at infinity), internal harmonics (irregular at the coordinate pole) are included in the expansion. This allows representation of the field from the central conductor in the TJ-II Heliac. The expansion is shown to provide a very accurate representation of the vacuum field in both cases. Algorithms for the accurate and rapid evaluation of the half-integer Legendre functions are provided.
\end{abstract}

\section{Introduction}

A good description of the magnetic field in vacuum is of importance in toroidal thermonuclear fusion devices. In vacuum, the Maxwell equations allow the introduction of a scalar potential, related to the magnetic field, that satisfies the Laplace equation. Several complete sets of solutions to the Laplace equations are known that allow expansions of the scalar potential (and therefore also of the magnetic field) in terms of moments [1], [2], [3], [4], [5]: Bessel functions (cylindrical coordinates), spherical harmonics (spherical coordinates) and toroidal harmonics (toroidal coordinates). The choice of coordinates and moments depends on the shape of the region of interest: a bad adaptation of the expansion functions to the region of interest will lead to a moment series of slow convergence. In toroidal devices such as stellarators or tokamaks, the most suitable expansion functions are toroidal harmonics. In literature, other (but related) types of expansions have been suggested because of the supposed difficulty in computing the toroidal harmonics [6], [7]. However, modern computational capacity and a moderately fast algorithm for evaluating these functions (see Appendix) remove this objection.

The advantages of the use of (toroidal) harmonics for field reconstruction are: (1) The toroidal harmonics constitute a complete and orthogonal set of solutions to the Laplace equation on a doubly connected toroidal region. This guarantees that regression procedures to determine the moments from magnetic field data will be stable and well-conditioned (when sufficient data are provided). The same cannot be said of some other systems of moments introduced in literature. (2) The toroidal harmonic moments can directly be interpreted as dipole, quadrupole, etc., field components. (3) When calculating a plasma equilibrium from external magnetic measurements, the moment formalism can be used for the calculation of the magnetic field outside the plasma, providing a systematic way to extrapolate inward from magnetic field pickup coil measurements towards the plasma boundary. Limiting the number of moments in this process is a robust way of suppressing measurement noise and stabilizing the extrapolation. The extrapolated field data close to or at the plasma boundary can then be used as input for a (fixed-boundary) equilibrium code. (4) The field of each external current-carrying coil can be expressed in terms of a number of moments (each moment scaling linearly with the coil current), so that for any combination of the external coils currents the external (vacuum) field 
can be computed in a fast way (no need to evaluate Green's functions) with high accuracy. (5) With a suitably designed set of pick-up coils, it becomes possible to separate the external (from field coils) and internal (from the plasma) contributions to the field, facilitating the analysis of the field caused by the plasma alone. (6) The magnetic field (in three dimensions) can be stored in a compact manner. Instead of storing the field components on a large number of grid points, only a limited number of moments needs to be stored, from which the field can be reconstructed.

\section{Expansion of the vacuum magnetic field in toroidal harmonics}

In vacuum, the magnetic field is subject to

$$
\nabla \cdot \boldsymbol{B}=0 \quad \text { and } \quad \nabla \times \boldsymbol{B}=0,
$$

from which follows

$$
\boldsymbol{B}=\nabla \psi \quad \text { and } \quad \Delta \psi=0 .
$$

Thus the scalar potential $\psi$ can be expanded in toroidal harmonics [1], [2].

We introduce toroidal coordinates [1], [8] (an alternative but equivalent definition is given in [4]). The relationship between the usual cylindrical coordinates $(R, \phi, Z)$ and toroidal coordinates $(\zeta, \eta, \phi)$ is as follows:

$$
R=R_{p} \frac{\sinh \zeta}{\cosh \zeta-\cos \eta}, \quad Z=R_{p} \frac{\sin \eta}{\cosh \zeta-\cos \eta}, \quad \phi=\phi,
$$

where $R_{p}$ is the pole of the coordinate system. Surfaces of constant $\zeta$ are tori with major radii $R=R_{p} / \tanh \zeta$ and minor radii $a=R_{p} / \sinh \zeta$. At $R=R_{p}, \zeta=\infty$, while at infinity and at $R=0, \zeta=0$. The coordinate $\eta$ is a poloidal angle and runs from 0 to $2 \pi$. See Appendix for the inverse transformation corresponding to Eq. (3).

In toroidal coordinates the Laplace operator becomes

$$
\begin{aligned}
\Delta= & \frac{(\cosh \zeta-\cos \eta)^{2}}{R_{p}^{2}}\left\{\frac{(\cosh \zeta-\cos \eta)}{\sinh \zeta} \frac{\partial}{\partial \zeta}\left(\frac{\sinh \zeta}{(\cosh \zeta-\cos \eta)} \frac{\partial}{\partial \zeta}\right)\right. \\
& \left.+(\cosh \zeta-\cos \eta) \frac{\partial}{\partial \eta}\left(\frac{1}{(\cosh \zeta-\cos \eta)} \frac{\partial}{\partial \eta}\right)+\frac{1}{\sinh ^{2} \zeta} \frac{\partial^{2}}{\partial \phi^{2}}\right]
\end{aligned}
$$

The equation $\Delta \psi=0$ separates with the substitution [1]:

$$
\psi=\sqrt{\cosh \zeta-\cos \eta} f(\zeta) g(\eta) h(\phi)
$$

and the separated equations are:

$$
\frac{1}{\sinh \zeta} \frac{\partial}{\partial \zeta}\left(\sinh \zeta \frac{\partial f}{\partial \zeta}\right)-\frac{n^{2} f}{\sinh ^{2} \zeta}-\left(m^{2}-\frac{1}{4}\right) f=0
$$




$$
\begin{aligned}
& \frac{\partial^{2} g}{\partial \eta^{2}}=-m^{2} g, \\
& \frac{\partial^{2} h}{\partial \phi^{2}}=-n^{2} h .
\end{aligned}
$$

A complete set of solutions to equation (6a) is provided by the half-integer Legendre functions or toroidal harmonics [1]. The solutions are singular at $\zeta=0$ or $\zeta=\infty$. Because the region $0<\zeta<\infty$ is doubly connected, two additional functions are needed in order to represent magnetic fields caused by net currents flowing along the $Z$-axis or along the pole, for these fields do not satisfy $\nabla \times \boldsymbol{B}=0$ on one-dimensional singularities outside the region $0<\zeta<\infty$. The scalar potential $\psi$ is therefore expanded as follows:

$$
\begin{aligned}
\psi & =M_{00}^{\phi} \psi_{\phi}+\delta_{\mathrm{int}} M_{00}^{\eta} \Psi_{\eta}+\sqrt{\cosh \zeta-\cos \eta} \\
& \times \sum_{m=0}^{M} \sum_{n=0}^{N}\left\{\left[M_{m n}^{c c e} \cos (n \phi) \cos (m \eta)+M_{m n}^{c s e} \cos (n \phi) \sin (m \eta)+M_{m n}^{s c e} \sin (n \phi) \cos (m \eta)\right.\right. \\
& \left.+M_{m n}^{s s e} \sin (n \phi) \sin (m \eta)\right] Q_{m-1 / 2}^{n}(\cosh \zeta) / Q_{m-1 / 2}^{n}\left(\cosh \zeta_{0}\right) \\
& +\delta_{\text {int }}\left[M_{m n}^{c c i} \cos (n \phi) \cos (m \eta)+M_{m n}^{c s i} \cos (n \phi) \sin (m \eta)\right. \\
& \left.\left.+M_{m n}^{s c i} \sin (n \phi) \cos (m \eta)+M_{m n}^{s s i} \sin (n \phi) \sin (m \eta)\right] P_{m-1 / 2}^{n}(\cosh \zeta) / P_{m-1 / 2}^{n}\left(\cosh \zeta_{0}\right)\right\}
\end{aligned}
$$

where the $M_{m n}^{x x x}$ are the moments. The $P_{m-1 / 2}^{n}$ and $Q_{m-1 / 2}^{n}$ are the half-integer Legendre functions [1], [2]. $\delta_{\text {int }}$ is a Kronecker symbol, the meaning of which is explained below. The term $M_{00}^{\phi} \psi_{\phi}$ is included to account for net currents along the $Z$-axis. The choice

$$
\psi_{\phi}=\phi
$$

generates an axisymmetric toroidal field $B_{\phi}=M_{00}^{\phi} / R$, corresponding to a line current $I=2 \pi \mu_{0}^{-1} M_{00}^{\phi} e_{Z}$ along the $Z$-axis. Likewise, the potential $\psi_{\eta}$ of Eq. (7a) generates a field $B=M_{00}^{\eta} \nabla \psi_{\eta}$ corresponding to a line current along the pole. The function $\psi_{\eta}$ can be written down as a series expansion, the leading term being proportional to $\eta$. However, a more compact representation is available in terms of the conjugate function $\chi[8]$ :

$$
\nabla \psi_{\eta}=R_{p} / R \nabla \chi \times e_{\phi}, \quad \chi=\frac{\sinh \zeta}{\sqrt{\cosh \zeta-\cos \eta}} P_{-1 / 2}^{1}(\cosh \zeta)
$$

The field thus generated is that of a line current $I=\left(\sqrt{2} \pi \mu_{0}\right)^{-1} M_{00}^{\eta} e_{\phi}$ along the torus axis (pole) $R=R_{p}, Z=0$.

The expansion functions are normalized with $Q_{m-1 / 2}^{n}\left(\cosh \zeta_{0}\right)$ and $P_{m-1 / 2}^{n}\left(\cosh \zeta_{0}\right)$ for numerical purposes: if the fixed surface $\zeta=\zeta_{0}$ is well-chosen (i.e. roughly centered in the region of interest), the expansion functions $Q_{m-1 / 2}^{n}(\cosh \zeta) / Q_{m-1 / 2}^{n}\left(\cosh \zeta_{0}\right)$ and $P_{m-1 / 2}^{n}(\cosh \zeta) / P_{m-1 / 2}^{n}\left(\cosh \zeta_{0}\right)$ will have values close to one in this region.

Comparing Eqs. (5) and (7), it should be clear that the expansion (7) is overcomplete: the moments $M_{m n}^{\text {sse } / i}$ depend in a non-linear way on $M_{m n}^{c c e / i}, M_{m n}^{c s e / i}$ and $M_{m n}^{s c e / i}$. However, the expansion (7) is preferable over other types of expansions that do not exhibit this overcompleteness, because it leads to a linear regression problem when determining the moments from a given field configuration. 
The expansion (7) can be simplified by taking account of symmetries imposed by the design of the machine. Tokamak symmetry (axisymmetric fields) implies, of course, that only $n=0$ terms appear in (7). Some other symmetries are:

$$
\begin{array}{ll}
\text { antisymmetry: } & \psi(R, Z, \phi)=-\psi(R,-Z,-\phi) \Rightarrow M_{m n}^{c c e / i}=M_{m n}^{s s e / i}=0, \\
\text { symmetry: } & \psi(R, Z, \phi)=+\psi(R,-Z,-\phi) \Rightarrow M_{m n}^{c s e / i}=M_{m n}^{s c e / i}=0 .
\end{array}
$$

Stellarators are antisymmetric (8a). The number of moments in the expansion is (note that $M_{0 n}^{s s e}=M_{0 n}^{s s i}=M_{0 n}^{s c e}=M_{0 n}^{s c i}=0$ for $\mathrm{n} \geq 0$ and $M_{m 0}^{s s e}=M_{m 0}^{s s i}=M_{m 0}^{c s e}=M_{m 0}^{c s i}=0$ for $m \geq 0$ ):

$$
N_{\text {moments }}=\left\lfloor\left(2 \frac{N}{N_{T}} M+\frac{N}{N_{T}}+M\right)(2-|s|)+2-\Theta(-s)\right\rceil\left(\delta_{\text {int }}+1\right),
$$

where $s=-1$ when (8a) applies, $s=1$ when (8b) applies and $s=0$ otherwise, and $\Theta(x)$ is the Heaviside function: $\Theta(x)=1$ when $x \geq 0$ and $\Theta(x)=0$ when $x<0$; and $\delta_{\text {int }}=0$ when the internal moments are not included in the expansion (7), $\delta_{\text {int }}=1$ otherwise. The toroidal periodicity $N_{T}$ of the machine (stellarator) implies that only toroidal moments $n=0, N_{T}, 2 N_{T}$, $\ldots, N$ are used. Absence of periodicity means $N_{T}=1$.

The expansion is only valid in a toroidally annular region between two $\zeta=$ constant surfaces where the current density is identically zero: say $\zeta=\zeta_{\text {in }}$ and $\zeta=\zeta_{\text {ex }}$, $\zeta_{\text {in }}>\zeta_{\text {ex }}$ (Fig. 1). The moments labeled " $i$ " are "internal moments" due to currents in the region $\zeta>\zeta_{\text {in }}$ (the "internal" or "plasma" region) and the moments labeled " $e$ " are "external moments" due to currents in the region $\zeta<\zeta_{\text {ex }}$ (the "external" or "coil" region). In the formalism, all currents, including magnetisation currents, are to be treated as true currents. If no current flows in the internal region (e.g. no plasma), all internal moments are equal to zero $\left(\delta_{\text {int }}=0\right)$.

It may be that some current-carrying coil enters one of the regions $\zeta>\zeta_{\text {in }}$ or $\zeta_{\text {ex }}<\zeta<$ $\zeta_{\text {in }}$; in the former case, the coil is conveniently represented by the internal moments (e.g. the central conductor of TJ-II, see Section 3), while in the latter case the coils can, strictly speaking, not be represented by the formalism and only the part of the field not due to these coils can be expanded in the harmonics (this occurs with the toroidal field coils of TJ-II, see section 3 ).

When $M$ and $N$ are sufficiently large, any function $\psi$ satisfying $\Delta \psi=0$ in the region of validity can in theory be approximated with arbitrary accuracy. The actual accuracy depends mainly on the accuracy with which the expansion functions (the Legendre polynomials) are known, and on the regression procedure (i.e. number and distribution of field data points to which the moment expansion is fitted, as well as their accuracy). The Appendix provides an algorithm for computing the Legendre functions.

The convergence of the expansion (7) depends critically on the choice of the pole $R_{p}$ when currents flow in the interior region (a plasma is present), the best choice being for the surface $\zeta=\zeta_{\text {in }}$ to coincide as closely as possible with the limiting surface of the currentcarrying region (plasma boundary). For example, if $R_{0}$ is the plasma major radius and a is the plasma minor radius, $R_{p}=\sqrt{R_{0}^{2}-a^{2}}$.

Using Eqs. $(2,3,7)$ the field can trivially be expressed in the moments $M_{m n}^{x x x}$. Specifically [1]:

$$
B=\nabla \psi=B_{R} e_{R}+B_{Z} e_{Z}+B_{\phi} e_{\phi},
$$

where the $e$ 's are the unit vectors of the coordinate system. The field components are: 


$$
\begin{aligned}
& B_{R}=\frac{\sinh \zeta}{R_{p}}\left(\frac{(1-\cosh \zeta \cos \eta)}{\sinh \zeta} \frac{\partial \psi}{\partial \zeta}-\sin \eta \frac{\partial \psi}{\partial \eta}\right), \\
& B_{Z}=\frac{\sinh \zeta}{R_{p}}\left(-\sin \eta \frac{\partial \psi}{\partial \zeta}-\frac{(1-\cosh \zeta \cos \eta)}{\sinh \zeta} \frac{\partial \psi}{\partial \eta}\right), \\
& B_{\phi}=\frac{(\cosh \zeta-\cos \eta)}{R_{p} \sinh \zeta} \frac{\partial \psi}{\partial \phi} .
\end{aligned}
$$

The contribution of the moment introduced in Eq. (7c) to the total field is found to be:

$$
\begin{aligned}
& B_{R}=\delta_{\mathrm{int}} M_{00}^{\eta} \frac{\sqrt{\cosh \zeta-\cos \eta} \sin \eta}{4 R_{p} \sinh \zeta}\left[\cosh \zeta P_{1 / 2}^{0}(\cosh \zeta)-P_{-1 / 2}^{0}(\cosh \zeta)\right], \\
& B_{z}=-\delta_{\mathrm{int}} M_{00}^{\eta} \frac{\sqrt{\cosh \zeta-\cos \eta}}{4 R_{p}}\left[\cos \eta P_{1 / 2}^{0}(\cosh \zeta)-P_{-1 / 2}^{0}(\cosh \zeta)\right], \\
& B_{\phi}=0 .
\end{aligned}
$$

Note that these expressions can also be written in cylinder coordinates in terms of the more familiar elliptic functions $K$ and $E$ [2].

For a magnetic field given on a number of grid points, the moments are determined by a regression using Eqs. (7) and (10) to find a linear expression of the field components in the moments, the latter being the regression coefficients.

\section{Application to stellarators}

As test cases for the potential of the moment formalism as presented in the previous sections, two stellarators were analysed. TJ-IU, an $l=1$ torsatron, and TJ-II, an $l=1$ heliac with central conductor, both under construction at the Asociación EURATOM-CIEMAT para Fusión. The fields corresponding to these machines were computed on a number of $N_{\text {grid }}$ points for standard configurations of coil currents, and the moments were determined by the regression procedure described in the previous section. The grid points were selected randomly within a specified region, in order to improve the regression and avoid aliasing effects. Then the field was reconstructed from the obtained moments, and the following two global error indicators were introduced:

The average error $\langle\varepsilon\rangle$ :

$$
<\varepsilon>=\frac{\sqrt{\left(\sum\left|B^{\text {rec }}-B^{\text {orig }}\right|^{2}\right) / N_{\text {grid }}}}{\left(\sum\left|B^{\text {orig }}\right|\right) / N_{\text {grid }}}
$$

and the maximum error $\varepsilon_{\max }$ : 


$$
\varepsilon_{\max }=\max \left(\frac{\left|B^{\text {rec }}-B^{\text {orig }}\right|}{\left|B^{\text {orig }}\right|}\right)
$$

Here $B^{\text {rec }}$ refers to the field as reconstructed using the expansion in toroidal harmonics, and $B$ orig to the original field as calculated from the coil currents using Biot-Savart's Law. The summation in Eq. (11a) and the maximum in Eq. (11b) are taken over all grid points. These quantities are well-defined because in the configurations we studied the $\boldsymbol{B}$-field did not vanish anywhere.

\subsection{Expansion of a TJ-IU standard configuration without plasma}

A standard TJ-IU Torsatron field configuration was analysed. TJ-IU has the following parameters: $l=1$, Toroidal periodicity $N_{T}=6$, major radius $R_{0}=0.6 \mathrm{~m}$, minor radius of the region of interest (the plasma region) $a_{\min }=0.15 \mathrm{~m}$, inner minor radius of the helical coil $a_{\text {coil }}=0.24 \mathrm{~m}$. The pole of the toroidal coordinate system is chosen at $R_{p}=0.55 \mathrm{~m}$, and $\cosh \left(\zeta_{0}\right)=5$. The stellarator symmetry is given by Eq. (8a) $(s=-1)$. We considered a toroidal region $0 \leq r \leq a_{\min }, 0 \leq \theta<2 \pi$ and $0 \leq \phi<2 \pi$, and selected 500 points randomly in this region for which the field was calculated from given external coil currents. The moments expansion of the field was fitted to these data. In the expansion (7) only the terms $n=0,6,12, \ldots, N$ were used because of the toroidal periodicity of $N_{T}=6$. Because no currents flow in the internal region (no plasma), all internal moments were set to $0\left(\delta_{\text {int }}=0\right)$. Fig. 2 shows the field reconstruction errors as defined by Eq. (6) for various choices of $N$ and $M$ for a standard configuration. It is observed that the errors decay exponentially to very low values, and that the regression remains stable even at very large numbers of moments, due to the orthogonality of the expansion functions. Fig. 3 shows Poincare plots for the exact magnetic field (as calculated from Biot-Savart's Law) and the approximate field as calculated form the moments for a few choices of $N$ and $M$. It is observed that the approximation improves as $N$ and $M$ increase, and that for $N=60, M=10$ there is no visible difference with the exact field, not only in the shape of the flux surfaces but even in the position of the individual points forming the Poincare plot. Finally, Fig. 4 shows the 1-profiles for the exact field and the approximate field for a few choices of $N$ and $M$. It is seen that the t-profile of the approximate field approaches the exact l-profile rapidly as $N$ and $M$ increase, and essentially coincides with the latter when $N=60$ and $M=10$.

\subsection{Expansion of a TJ-II standard configuration without plasma}

The stellarator TJ-II is a heliac with a central conductor with $l=1$ and toroidal periodicity $N_{T}=4$, major radius $R_{0}=1.5 \mathrm{~m}$ [9]. The stellarator symmetry is given by Eq. (8a) $(s=-1)$. As mentioned in section 2, the topology of the 32 circular toroidal field (TF) coils ( 8 per period) does not permit, in theory, the definition of a region of validity as with TJ-IU, because of their helical swing. The TF coils are circular with a radius of $0.425 \mathrm{~m}$, and the centre $R_{c}(\phi), Z_{c}(\phi)$ of each coil is displaced by a distance $r_{\text {swing }}=0.2825 \mathrm{~m}$ from the major axis $R=R_{0}, Z=0$, according to the law $R_{\mathrm{c}}=R+r_{\text {swing }} \cos \left(N_{T} \phi\right)$ and $Z_{\mathrm{c}}=-r_{\text {swing }} \sin \left(N_{T} \phi\right)$. The field generated by the TF coils is mainly toroidal, although some radial and vertical fields exist in the interstitial coil regions (i.e. field ripple) which is mainly of importance near the coils and is small in the region of interest, well inside the TF coils. Therefore, modelling each TF coil with one which has a larger radius at the same position (carrying a correspondingly larger 
current in order to generate the same toroidal field) will not affect the field in the region of interest much; more specifically, this procedure does not affect the average toroidal field at all, but it neglects the field ripple. Thus, computing the moments assuming that the TF coils have a sufficiently large radius such that they do not invade the region $\zeta_{\mathrm{ex}}<\zeta<\zeta_{\text {in }}$ (although in reality they do) should be able to give a reasonable reproduction of the total magnetic field, except perhaps for the field ripple. We investigate this in the following.

We considered a helical region that remained within the TF coils everywhere. In each plane $\phi=$ constant, the boundary of the region is an ellipse with short half axis $a=0.175 \mathrm{~m}$ and long half axis $b=\varepsilon a$, where the ellipticity is $\varepsilon=1.4$. The ellipse is centered on $R_{\mathrm{e}}(\phi), Z_{\mathrm{e}}(\phi)$

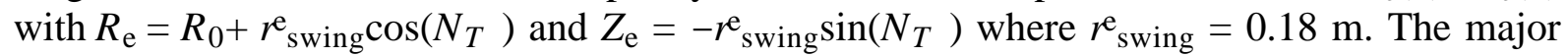
axis $\mathrm{b}$ of the ellipse is perpendicular to the direction $\left(R_{\mathrm{e}}-R_{0}, Z_{\mathrm{e}}\right)$. Finally, points less than a distance $0.13 \mathrm{~m}$ from the major axis are excluded from the region. This elliptical-helical region with a gap is designed to approximately enclose a typical plasma. The pole of the toroidal coordinate system is chosen at $R_{\mathrm{p}}=1.5 \mathrm{~m}$, and $\cosh \left(\zeta_{0}\right)=5.7$. We selected 1000 points randomly in this region for which the field was calculated from given external coil currents. The moments expansion of the field was fitted to these data. In the expansion (7) only the terms $\mathrm{n}=$ $0,4,8, \ldots, N$ were used because of the toroidal periodicity of $N_{T}=4$. To represent the field generated by the central conductor, the internal moments were used $\left(\delta_{\text {int }}=1\right)$. Fig. 5 displays the field reconstruction errors for various choices of $N$ and $M$ for a standard configuration. Again, it is observed that the errors decay exponentially to very low values and that the regression remains stable even at very large numbers of moments. Also, it is seen that the inclusion of moments with $n=32$ leads to an additional reduction of the reconstruction error (over the exponential decay), because that allows representation of the field ripple (recall that there are $32 \mathrm{TF}$ coils). Further it is seen that the presence of the TF coils in the region of validity does not affect the accuracy much, probably because of the reason given earlier: they may be replaced by an equivalent set of coils not invading the region of validity but generating essentially the same field in the helical region of interest as defined above. Fig. 6 shows Poincaré plots for the exact magnetic field (as calculated from Biot-Savart's Law) and the approximate field as calculated form the moments for a few choices of $N$ and $M$. It is observed that the approximation improves as $N$ and $M$ increase, and that for $N=48, M=12$ there is only some slight difference with the exact field left. For $N=60, M=15$ the field errors as defined above are reduced (see Fig. 5) but the Poincaré plot shows that, although the interior flux surfaces are reproduced with excellent precision, the outermost flux surface suffers some small higher-harmonic deformation, due to overfitting. Finally, Fig. 7 shows the 1-profiles for the exact field and the approximate field for a few choices of $N$ and $M$. It is seen that the 1-profile of the approximate field approaches the exact l-profile rapidly as $N$ and $M$ increase.

\section{Future extensions of this work}

A first extension of the work presented here is to calculate the moments in the presence of a plasma from the field in the vacuum region surrounding the plasma. It is to be foreseen that this will cause no difficulties in the TJ-IU case, since the plasma can simply be represented by the internal moments. In the case of TJ-II, however, due to the helical shape of the plasma and the presence of the central conductors, this may be not so simple and probably only the part of the vacuum field on the outside of the plasma (i.e. on the side away from the central conductor) can be represented by toroidal harmonics.

In the case of TJ-IU and perhaps also in the case of TJ-II we will then create a small database containing some scans of the most important plasma parameters and the response of the moments. This can be used to create a bigger database by interpolating between equilibria in 
moments-space, which may then be used in statistical analyses to determine the response of (magnetic) diagnostics to plasma parameters (this will aid data interpretation). It is even foreseen to use this bigger database to design the magnetic diagnostic in such a way that it provides maximum information on some selected plasma parameters.

\section{Conclusions and comparison with other systems of moments}

Expanding the vacuum magnetic field in stellarators (or tokamaks) in toroidal harmonics is a successful method for representing the field in a compact manner. The toroidal harmonics or half-integer Legendre functions form a complete and orthogonal set of expansion functions, which allows determination of the moments with very good precision that is only limited by computational accuracy. Other expansion functions have been suggested in literature for this problem, but all seem to suffer from some drawbacks compared to the system of moments discussed here. The cylindrical expansion functions with a first-order toroidal correction as suggested in [7] are complete nor orthogonal, and consequently the reconstruction error $\langle\varepsilon\rangle$ does not drop to very low values (in [7], $\langle\varepsilon\rangle=0.46 \%$ is mentioned as best result for a standard ATF configuration without plasma, but it should be mentioned that only a very small number of moments was used). The Dommaschk potentials [6], which are both orthogonal and complete for fields regular at the torus axis, have the advantage that they are easy to evaluate, but, as noted in [6], they fail to represent Heliac fields with singularities at the toroidal axis, because they do not contain the equivalent of the internal moments as described in this paper. We have demonstrated that the half-integer Legendre functions can even approximate vacuum Heliac fields with high accuracy (the TJ-II case).

It has been said many times that the half-integer legendre functions are difficult to evaluate (e.g. [6]). In the Appendix to this paper we provide an analysis of this supposed difficulty and a solution. We do not believe that the numerical evaluation of the half-integer Legendre functions is an obstacle to their use.

\section{Acknowledgements}

This work was made possible by Commission of the European Communities Bursary ERB4001GT921485. The authors would like to thank the TJ-IU and TJ-II teams at the Asociación EURATOM-CIEMAT para Fusión for a very pleasant cooperation. 


\section{Appendix}

\section{A.1 Toroidal coordinates inverse transformation}

The transformation from cylindrical coordinates $(R, Z, \phi)$ to toroidal coordinates $(\zeta, \eta, \phi)$ (the inverse of Eq. (3)) is carried out as follows:

$$
\tanh \zeta=2\left(\frac{R}{R_{p}}+\frac{Z^{2}}{R R_{p}}+\frac{R_{p}}{R}\right)^{-1}, \quad \tan \eta=\frac{Z \sinh \zeta}{R \cosh \zeta-R_{p} \sinh \zeta}
$$

\section{A.2 Evaluation of the half-integer Legendre functions}

In this Appendix, we discuss an algorithm to evaluate the half-integer Legendre functions or toroidal harmonics, since there are no standard computer library routines available for these functions. The half-integer Legendre functions can be expressed in terms of the Hypergeometric function $\left.F(a, b ; c ; z)={ }_{2} F_{1} a, b ; c ; z\right)[1,2]$ (Note an unimportant difference of a factor $(-1)^{n}$ between the $Q_{m-1 / 2}^{n}$ 's and of a factor $(\sqrt{-1})^{n}$ between the $P_{m-1 / 2}^{n}$ 's in the two cited references, as discussed in [5]. We adopt the normalization of [2]):

$$
\begin{aligned}
& P_{m-1 / 2}^{n}(\cosh \zeta)=\frac{\Gamma\left(m+n+\frac{1}{2}\right)}{2^{n} n ! \Gamma\left(m-n+\frac{1}{2}\right)} \tanh ^{n} \zeta \cosh ^{m-1 / 2} \zeta \\
& \times F\left[\frac{1}{2}\left(n-m+\frac{1}{2}\right), \frac{1}{2}\left(n-m+\frac{3}{2}\right) ; n+1 ; \tanh ^{2} \zeta\right] \\
& Q_{m-1 / 2}^{n}(\cosh \zeta)=\frac{\Gamma\left(\frac{1}{2}\right) \Gamma\left(m+n+\frac{1}{2}\right)}{2^{m+1 / 2} \Gamma(m+1)} \frac{\tanh ^{n} \zeta}{\cosh ^{m+1 / 2} \zeta} \\
& \times F\left[\frac{1}{2}\left(m+n+\frac{1}{2}\right), \frac{1}{2}\left(m+n+\frac{3}{2}\right) ; m+1 ; \frac{1}{\cosh ^{2} \zeta}\right\rceil
\end{aligned}
$$

where $\Gamma(x)$ is the Gamma function.

The Hypergeometric function $F(a, b ; c ; z)$ can be expanded as a series (note that here $a$, $b, c, z$ are all real and $0<z<1)[2]$ :

$$
F(a, b ; c ; z)=\sum_{i=0}^{\infty} S(i), \quad \text { with } S(i)=\frac{(a+i-1)(b+i-1)}{(c+i-1)} \frac{z}{i} S(i-1), \mathrm{i} \geq 1, \text { and } S(0)=1 .
$$

Convergence is discussed in [2]. If this series is truncated at some number $i=i_{\text {trunc }}$, $i_{\text {trunc }} \gg \max (1,|a|,|b|,|c|)$, the absolute error $\varepsilon$ in $F(a, b ; c ; z)$ is estimated by

$$
\varepsilon\left(i_{\text {trunc }}\right)=S\left(i_{\text {trunc }}\right) \frac{z}{1-z}
$$

Thus it is seen that truncation errors are important for $P_{m-1 / 2}^{n}(\cosh \zeta)$ when $\zeta$ is large (near the pole of the coordinate system), and for $Q_{m-1 / 2}^{n}(\cosh \zeta)$ when $\zeta$ is close to 0 . The series is evaluated ( $i_{\text {trunc }}$ incremented) until $\varepsilon_{\text {rel }}\left(i_{\text {trunc }}\right)$ falls below some preset relative precision $\delta$ : 


$$
\varepsilon_{\text {rel }}\left(i_{\text {trunc }}\right)=\left|\varepsilon\left(i_{\text {trunc }}\right)\left(\sum_{i=0}^{i_{\text {rrunc }}} S(i)\right)^{-1}\right|<\delta
$$

or when $i_{\text {trunc }}$ reaches some preset maximum $N_{\text {trunc }}$. A best estimate for $F(a, b ; c ; z)$ from the truncated series, with the condition that $i_{\text {trunc }} \gg \max (1,|a|,|b|,|c|)$, is then given by:

$$
F(a, b ; c ; z) \approx \sum_{i=0}^{i_{\text {trunc }}} S(i)+\varepsilon\left(i_{\text {trunc }}\right)
$$

(we only use this estimate when $i_{\text {trunc }}$ reaches $N_{\text {trunc }}$ ).

It turns out that for values of the argument in the range $2 \leq \cosh \zeta \leq 15$ (this range amply covers the requirements we have encountered in practice), the hypergeometric series corresponding to $Q_{m-1 / 2}^{n}$ converges rapidly ( $i_{\text {trunc }} \leq 73$ for a wide range of $n$ - and $m$-values, i.e. $0 \leq n \leq 60$ and $0 \leq m \leq 60$, when the termination criterion is set to $\delta=10^{-10}$ ), whereas the series corresponding to $P_{m-1 / 2}^{n}$ converges much slower $\left(i_{\text {trunc }} \leq 5247\right.$ under the same conditions). Note, however, that the convergence of the series corresponding to $P_{m-1 / 2}^{n}$ improves as $m$ increases ( $i_{\text {trunc }} \leq 985$ for $m>10$ under the above conditions). Note, also, that these values of $i_{\text {trunc }}$ are maxima taken over the whole range of $\zeta_{-}, n$ - and $m$-values as specified; in general the series converge much faster (e.g. $i_{\text {trunc }}$ is of the order of 10 in most cases for $\left.Q_{m-1 / 2}^{n}\right)$. Finally, recall that the $P_{m-1 / 2}^{n}$ are only required when internal field sources are present (cf. Eq. (7)).

Evaluation of $Q_{m-1 / 2}^{n}$ and $P_{m-1 / 2}^{n}$ for a range of $m$-values for fixed $n$ and $\zeta$ can be done more efficiently using the recurrence relations [2]:

$$
\begin{aligned}
& P_{m-1 / 2}^{n}=\left(m-n+\frac{1}{2}\right)^{-1}\left(2 m \cosh \zeta P_{m-1 / 2}^{n}-\left(m+n-\frac{1}{2}\right) P_{m-3 / 2}^{n}\right), \\
& Q_{m-3 / 2}^{n}=\left(m+n-\frac{1}{2}\right)^{-1}\left(2 m \cosh \zeta Q_{m-1 / 2}^{n}-\left(m-n+\frac{1}{2}\right) Q_{m+1 / 2}^{n}\right) .
\end{aligned}
$$

These relations should be used in the manner as suggested by Eqs. (A.3) and (A.4), i.e. with ascending $m$ for $P_{m-1 / 2}^{n}$ and descending $m$ for $Q_{m-1 / 2}^{n}$, in order to retain the largest possible number of significant digits in the results.

Computation time may be further reduced by introducing the following approximation:

$$
\begin{aligned}
\log \left(\left|P_{m-1 / 2}^{n}(\cosh \zeta)\right|\right)=\alpha_{0}^{n m} & +\alpha_{1}^{n m} \log (\cosh \zeta)+\alpha_{2}^{n m} \frac{\cosh \zeta}{\cosh \zeta_{n o r m}} \\
& +\cdots+\alpha_{k}^{n m}\left(\frac{\cosh \zeta}{\cosh \zeta_{n o r m}}\right)^{k-1}+\cdots
\end{aligned}
$$

where the coefficients $\alpha_{k}^{n m}$ are found from a least-squares fit to the Legendre functions as calculated using the method described above, and are stored in a file for later use. The same type of expansion is also used to find approximations to $Q_{m-1 / 2}^{n}(\cosh \zeta)$. Note that the expansion ignores the sign of $P_{m-1 / 2}^{n}$ and $Q_{m-1 / 2}^{n} . P_{m-1 / 2}^{n}$ is negative if and only if $m<n$ and $m+n=$ odd, while $Q_{m-1 / 2}^{n}$ is always positive. We found that for $2 \leq \cosh \zeta \leq 15,0 \leq n \leq 60$ and $0 \leq m \leq 60$, taking $\cosh \zeta_{\text {norm }}=5.7$ and truncating this expansion at $k=16$, the average and maximum relative errors in the calculation of $P_{m-1 / 2}^{n}$ are $\varepsilon_{r e l}^{P}(a v)=4 \times 10^{-5}$ and $\varepsilon_{r e l}^{P}(\max )=1 \times 10^{-3}$, and similar numbers for $Q_{m-1 / 2}^{n}$. The error in the series approximation of $P_{m-1 / 2}^{n}$ and $Q_{m-1 / 2}^{n}$ increases almost linearly with $n$. We achieved an average acceleration of a 
factor 24 for $2 \leq \cosh \zeta \leq 15,0 \leq n \leq 60$ and $0 \leq m \leq 60$ : a call to compute both $P_{m-1 / 2}^{n}$ and $Q_{m-1 / 2}^{n}$ lasted on average $102 \mu \mathrm{s}$ instead of $2.42 \mathrm{~ms}$ on a Cray YMP, while suffering only a moderate loss of accuracy in the calculation of magnetic fields (a factor of 2 in a particular case). The results presented in this paper we obtained without using this expansion, since computing time was not a problem. However, we re-computed the results presented in Section 3.1 (corresponding to the TJ-IU torsatron) with the approximations as described here, and observed no detectable difference in any of the Poincare plots, while the calculated $\imath$ values differed only in the sixth significant digit.

Finally, to evaluate Eq. (10), the derivatives of the Legendre functions are needed [2]:

$$
\frac{\partial Q_{m-1 / 2}^{n}(\cosh \zeta)}{\partial \zeta}=\frac{\left(m-\frac{1}{2}\right)}{\tanh \zeta} Q_{m-1 / 2}^{n}(\cosh \zeta)-\frac{\left(n+m-\frac{1}{2}\right)}{\sinh \zeta} Q_{m-3 / 2}^{n}(\cosh \zeta)
$$

and the same for the $P_{m-1 / 2}^{n}$. Note that $Q_{m-1 / 2}^{n}$ and $P_{m-1 / 2}^{n}$ are invariant under the replacement $m \rightarrow-m$ [4], [5]. 


\section{References}

[1] Morse and Feshbach, Methods of theoretical physics, McGraw-Hill, New York, 1953

[2] Abramowitz and Stegun, Handbook of mathematical functions, Dover, New York, 1972

[3] K. Miyamoto, Recent stellarator research, Nucl. Fusion 18 (1978) 243 - 284

[4] J.R. Cary, Construction of three-dimensional vacuum magnetic fields with dense nested flux surfaces, Phys. Fluids 27 (1984) 119 - 128

[5] B.J. Braams, Computational studies in tokamak equilibrium and transport, Thesis, FOM-Institute "Rijnhuizen", Nieuwegein, The Netherlands (1986)

[6] W. Dommaschk, Representations for vacuum potentials in stellarators, Comp. Phys. Comm. 40 (1986) 203-218

[7] G.H. Neilson, J.H. Harris, Harmonic analysis for magnetic configuration control in experimental stellarator devices, Nucl. Fusion 27 (1987) 711-724

[8] F. Alladio, F. Chrisanti, Analysis of MHD equilibria by toroidal multipolar expansions, Nucl. Fusion 26 (1986) 1143

[9] C. Alejaldre, J.J. Alonso Gonzalo, J. Botija Perez, et al., TJ-II project: a flexible heliac stellarator, Fusion Techn. 17 (1990) 131-139 
Figures

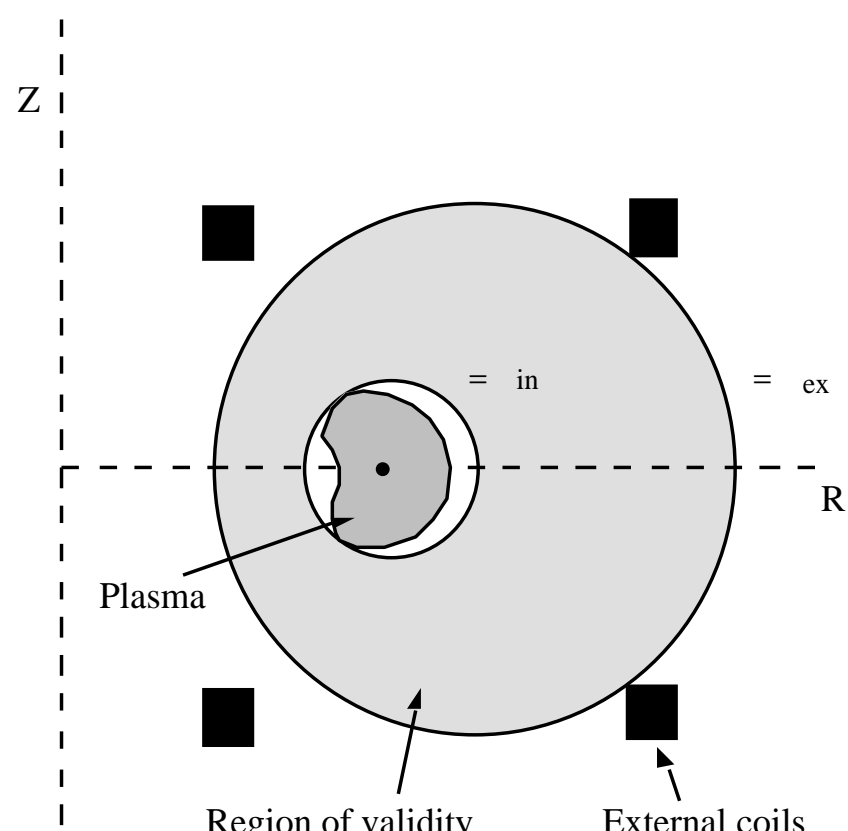

Fig. 1 Region of validy of the expansion of the vacuum magnetic field in toroidal harmonics. The region is delimited by two coordinate surfaces such that $\zeta_{\text {ex }}<\zeta<$ $\zeta_{\text {in }}$. Within this region the current density is identically zero. Exceptions to the latter statement are discussed in the text.

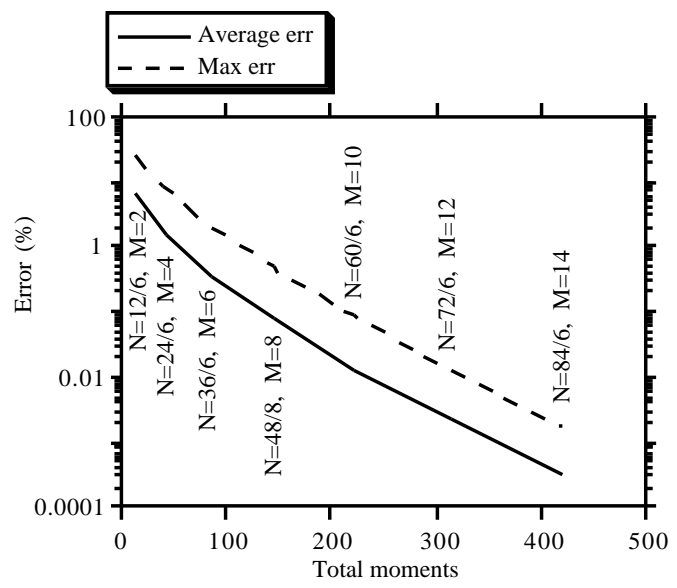

Fig. 2 Field reconstruction errors as defined by Eq. (11) for various choices of $N$ and $M$ for a TJ-IU standard configuration. The errors decay exponentially to very low values. 
Exact, $\phi=0^{\circ}$

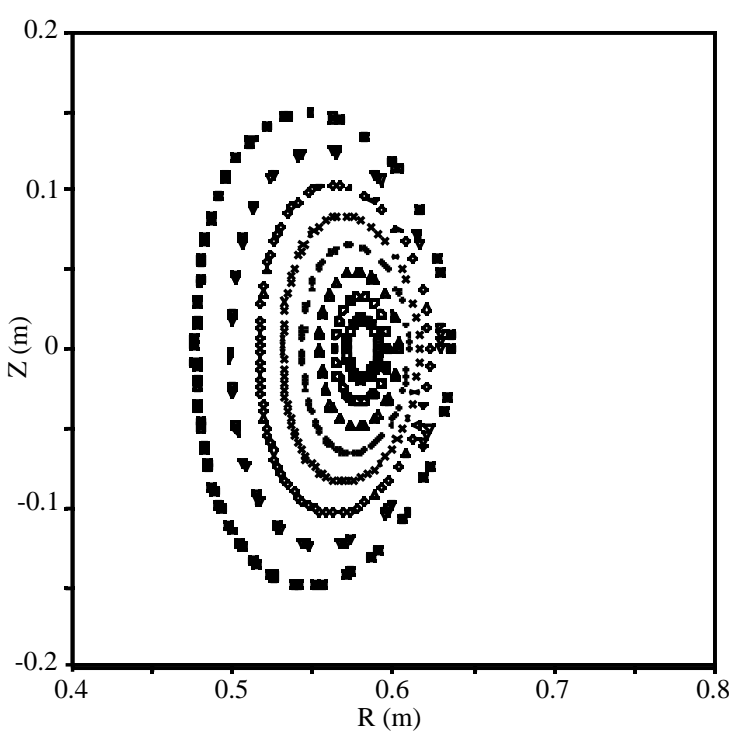

Exact, $\phi=15^{\circ}$

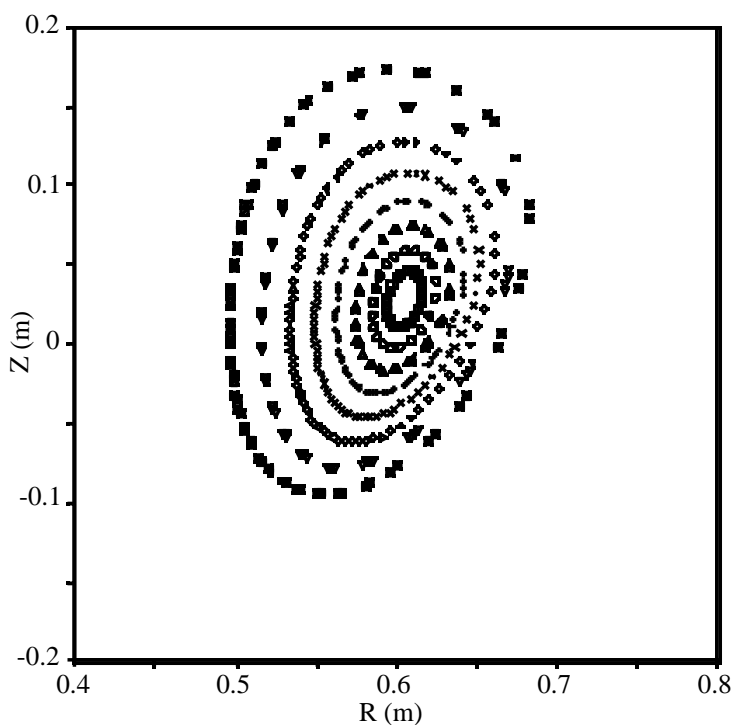

Fig. 3a Poincaré plots for the exact magnetic field of a TJ-IU standard configuration (as calculated from Biot-Savart's Law) at $\phi=0^{\circ}$ and $\phi=15^{\circ}$. Note that the third and seventh surfaces (counting outwards) are close to the resonance $l=n / m=6 / 20$.

$\mathrm{N}=24, \mathrm{M}=4, \phi=0^{\circ}$

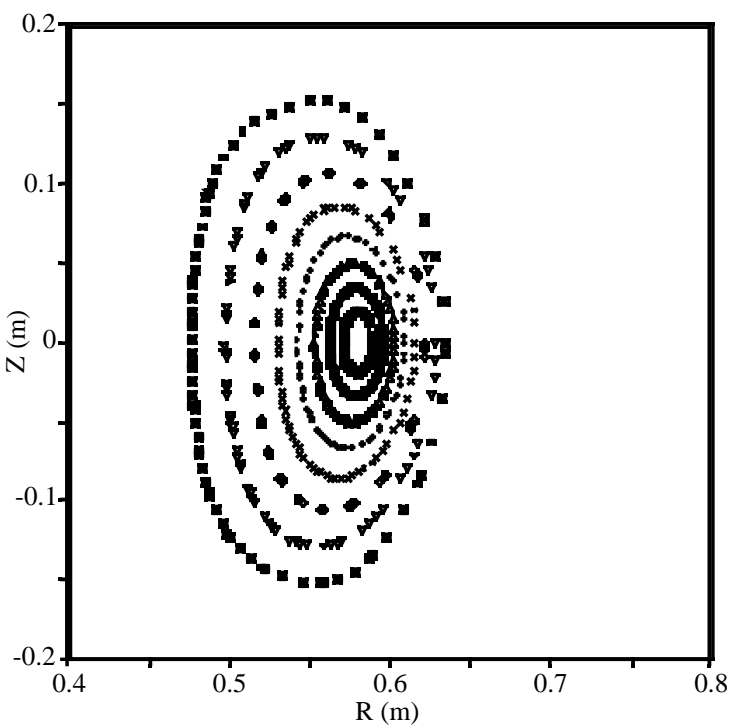

$\mathrm{N}=24, \mathrm{M}=4, \phi=15^{\circ}$

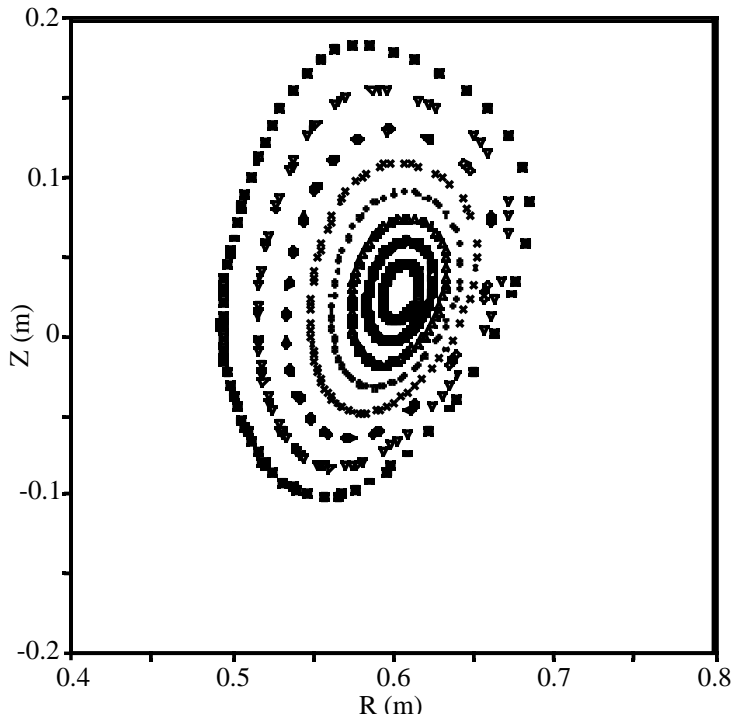

Fig. $3 \mathrm{~b}$ Poincare plots for the approximate magnetic field corresponding to the TJ-IU standard configuration as shown in Fig. 3a, using an expansion in toroidal harmonics with $N=24$ and $M=4$, at $\phi=0^{\circ}$ and $\phi=15^{\circ}$. Strong deformation of the flux surfaces w.r.t. Fig. $3 \mathrm{a}$ is observed. 

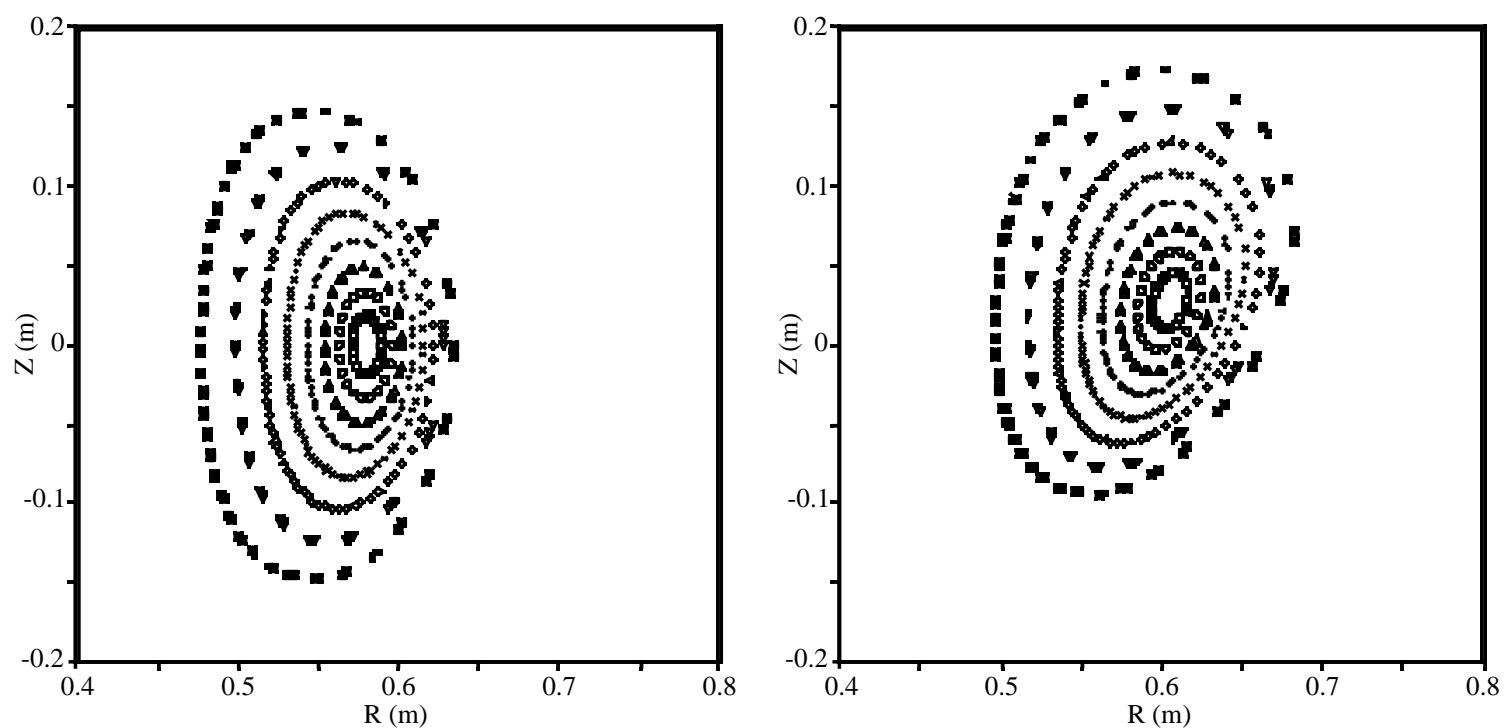

Fig. 3c Poincare plots for the approximate magnetic field corresponding to the TJ-IU standard configuration as shown in Fig. 3a, using an expansion in toroidal harmonics with $N=48$ and $M=8$, at $\phi=0^{\circ}$ and $\phi=15^{\circ}$. The shape of the flux surfaces corresponds closely to the shape of the case shown in Fig. 3a, but the individual points in the plots do not correspond (indicating a significant difference in the 1-profile).

$\mathrm{N}=60, \mathrm{M}=10, \phi=0^{\circ}$

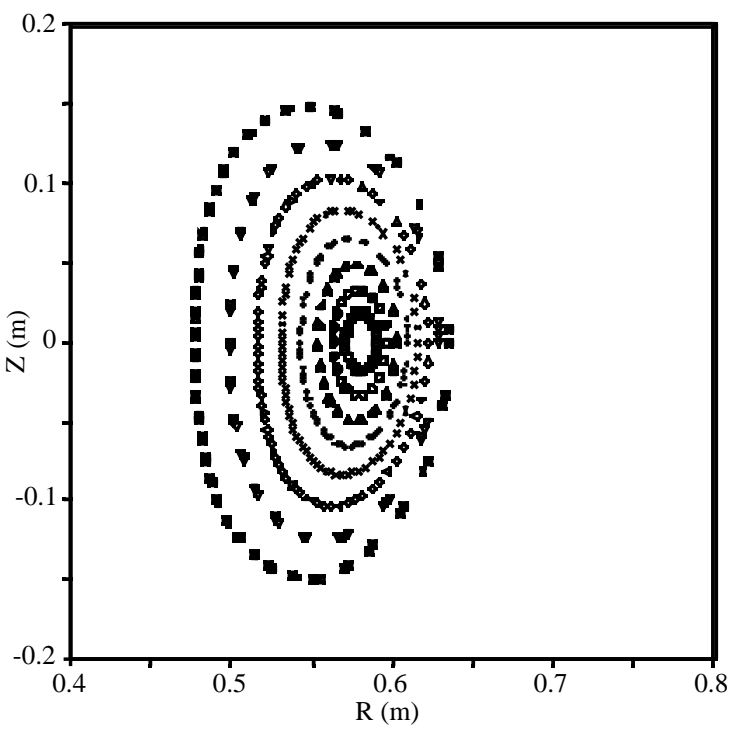

$\mathrm{N}=60, \mathrm{M}=10, \phi=15^{\circ}$

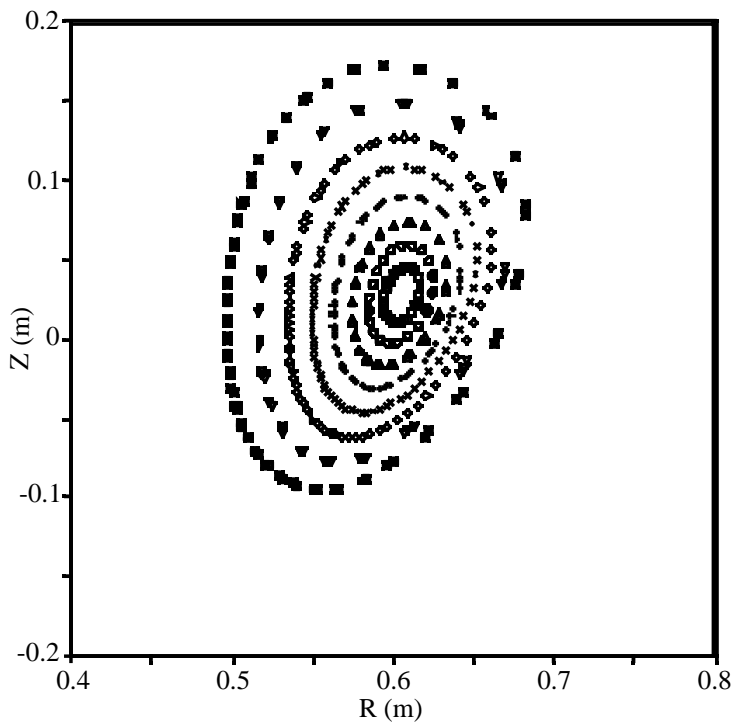

Fig. 3d Poincaré plots for the approximate magnetic field corresponding to the TJ-IU standard configuration as shown in Fig. 3a, using an expansion in toroidal harmonics with $N=60$ and $M=10$, at $\phi=0^{\circ}$ and $\phi=15^{\circ}$. No difference w.r.t. Fig. $3 \mathrm{a}$ is detectable. 


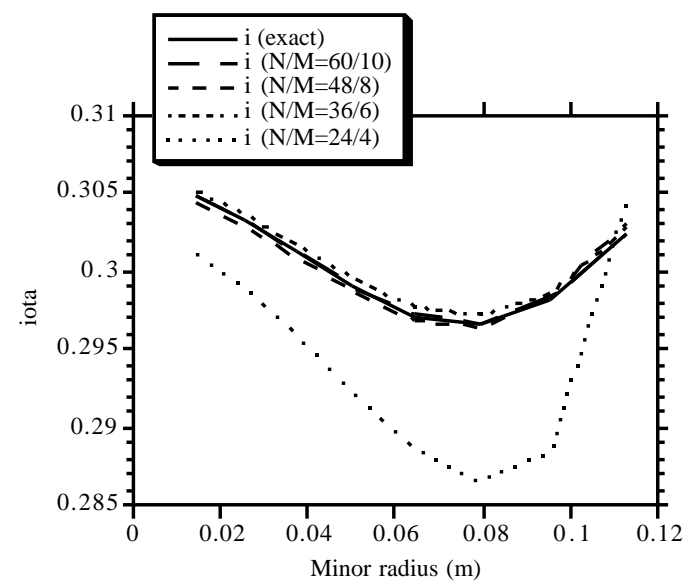

Fig. 4 1-profiles for the exact field and the approximate field for a few choices of $N$ and $M$, for a standard configuration of TJ-IU. It is seen that the l-profile of the approximate field approaches the exact $\mathrm{l}$-profile rapidly as $N$ and $M$ increase, and essentially coincides with the latter when $N=60$ and $M=10$.

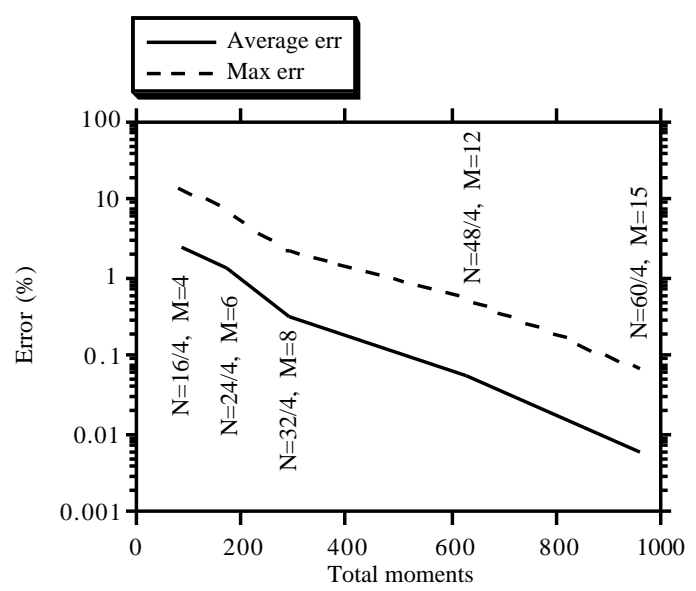

Fig. 5 Field reconstruction errors as defined by Eq. (11) for various choices of $N$ and $M$ for a TJ-II standard configuration. The errors decay exponentially to low values. 

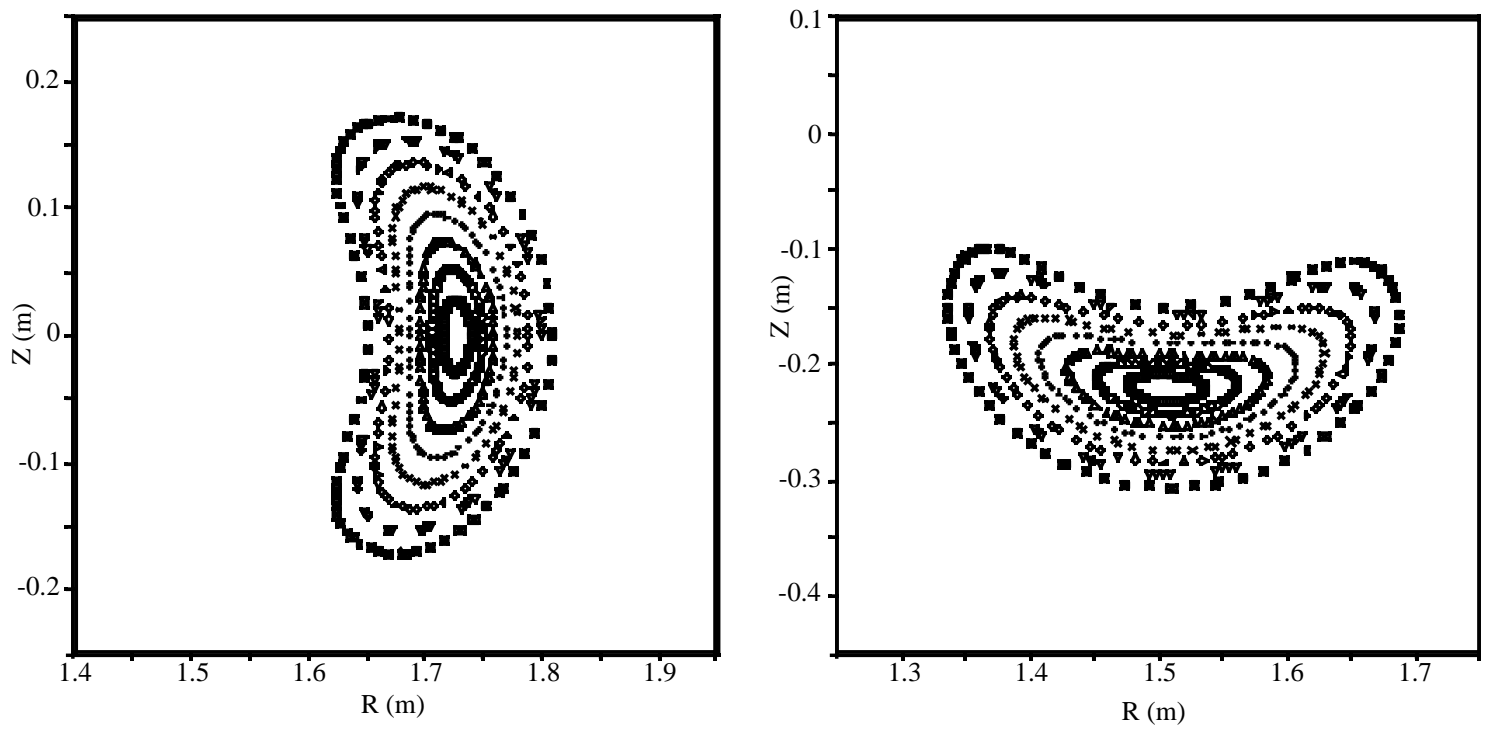

Fig. 6a Poincaré plots for the exact magnetic field of a TJ-II standard configuration (as calculated from Biot-Savart's Law) at $\phi=0^{\circ}$ and $\phi=22.5^{\circ}$. Note that the outermost surface but one is close to the resonance $l=n / m=28 / 19$.
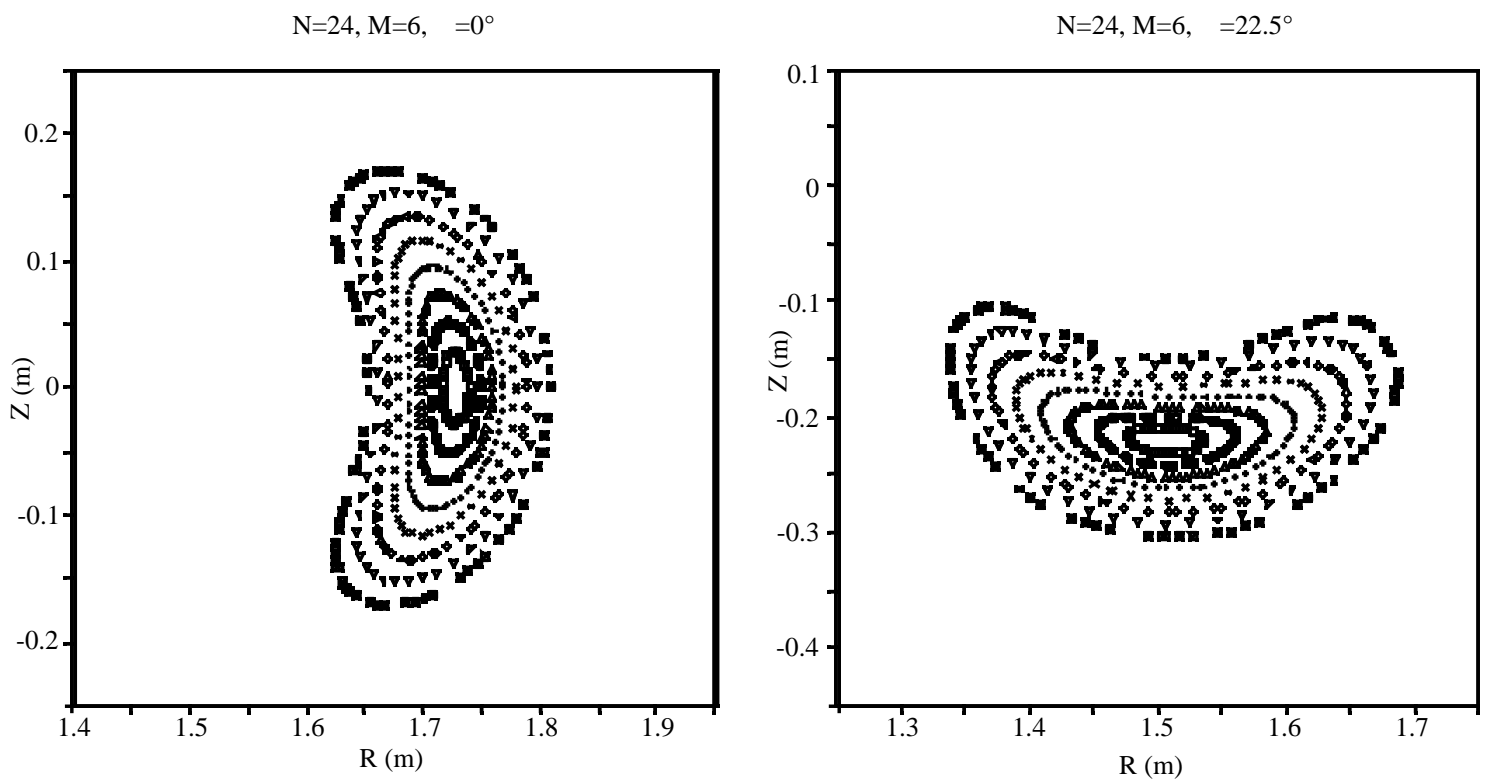

Fig. 6b Poincaré plots for the approximate magnetic field corresponding to the TJ-II standard configuration as shown in Fig. 3a, using an expansion in toroidal harmonics with $N=24$ and $M=6$, at $\phi=0^{\circ}$ and $\phi=22.5^{\circ}$. Slight deformation of the flux surfaces w.r.t. Fig. $6 \mathrm{a}$ is observed, and the outermost surface but one is seen not to be close to resonance. 

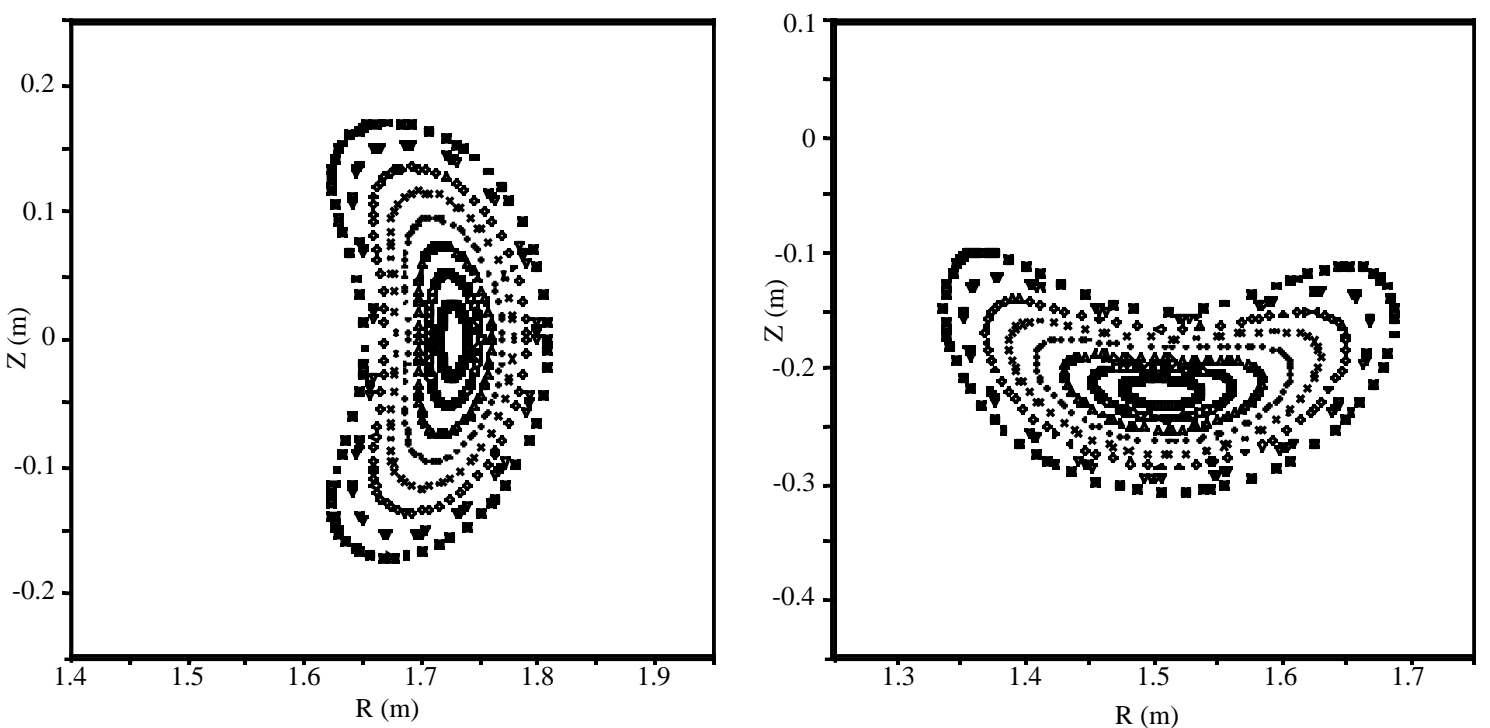

Fig. 6c Poincaré plots for the approximate magnetic field corresponding to the TJ-II standard configuration as shown in Fig. 3a, using an expansion in toroidal harmonics with $N=48$ and $M=12$, at $\phi=0^{\circ}$ and $\phi=22.5^{\circ}$. The shape of the flux surfaces corresponds rather closely to the shape of the case shown in Fig. 6 a.

$\mathrm{N}=60, \mathrm{M}=15, \phi=0^{\circ}$

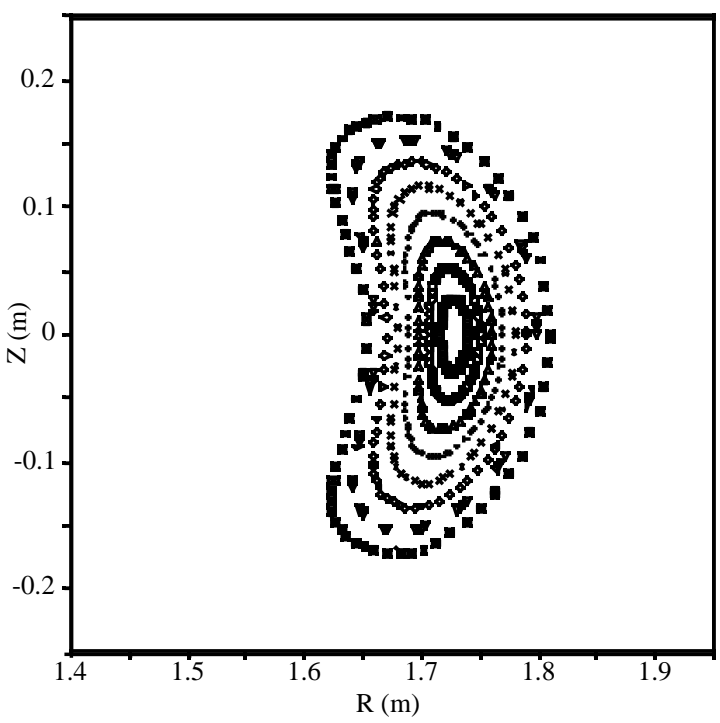

$\mathrm{N}=60, \mathrm{M}=15, \phi=22.5^{\circ}$

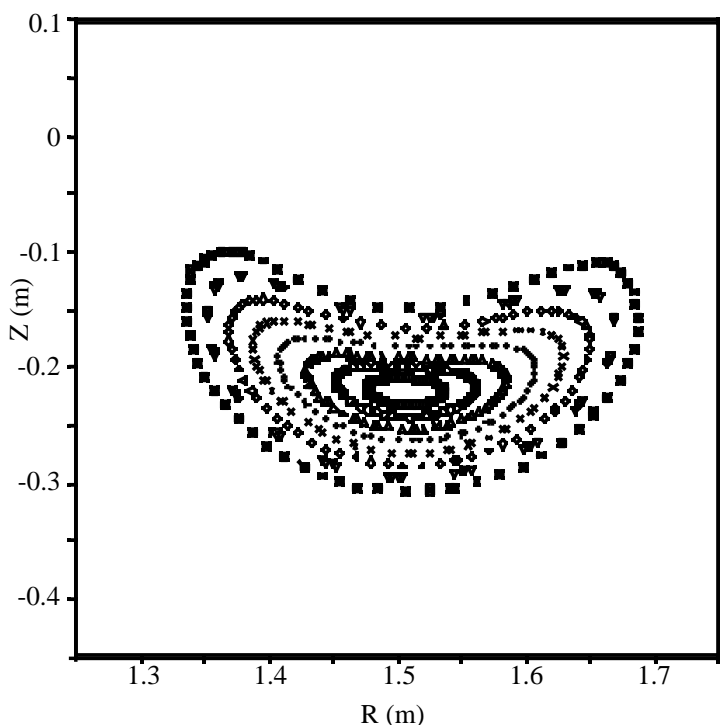

Fig. 6d Poincaré plots for the approximate magnetic field corresponding to the TJ-II standard configuration as shown in Fig. 3a, using an expansion in toroidal harmonics with $N=60$ and $M=15$, at $\phi=0^{\circ}$ and $\phi=22.5^{\circ}$. There is a slight improvement w.r.t. the plots of Fig. 6c. 


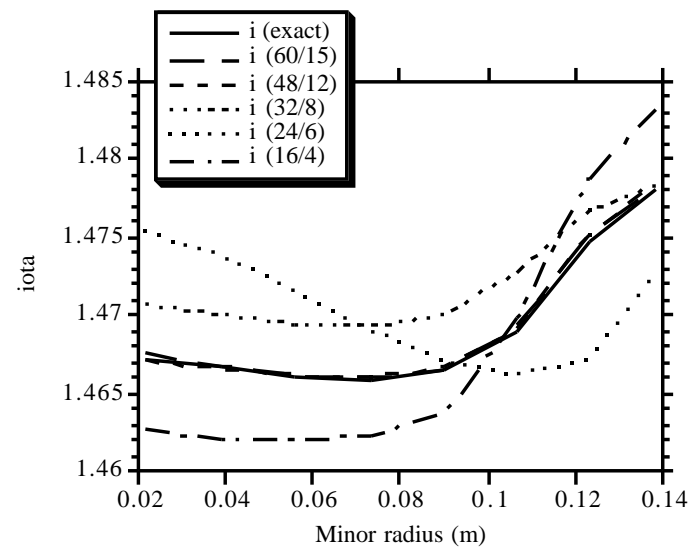

Fig. 7 1-profiles for the exact field and the approximate field for a few choices of $N$ and $M$, for a standard configuration of TJ-II. It is seen that the 1-profile of the approximate field approaches the exact $\mathrm{l-profile}$ rapidly as $N$ and $M$ increase, and is very close the latter when $N=48$ and $M=12$ or $N=60$ and $M=15$. 


\section{Erratum}

The following sentence (following Eq. (7c)):

The field thus generated is that of a line current $I=\left(\sqrt{2} \pi \mu_{0}\right)^{-1} M_{00}^{\eta} e_{\phi}$ along the torus axis (pole) $R=R_{p}, Z=0$.

should be replaced by:

The field thus generated is that of a line current $I=\left(\sqrt{2} / \mu_{0}\right) M_{00}^{\eta} e_{\phi}$ along the torus axis (pole) $R=R_{p}, Z=0$. 